$\mathrm{D}$ ie Fremdsprachenforschung steht im engen Zusammenhang mit einer wachsenden interkulturellen Verständigung und einer internationalen Zusammenarbeit, um nicht zuletzt von einer Internationalisierung der Welt zu sprechen. Interkulturelle Kommunikation, interkulturelles Lernen und interkulturelles Verstehen haben an Bedeutung gewonnen.

Für interkulturelles Lernen folgt, dass Lernenden entsprechende interkulturelle Lernangebo-

\section{Jens Drewitz}

\section{Goethe-Institut}

Aussenstelle Granada te geboten werden, um so eine kulturelle Begegnung zu sichern, zu fördern und zu entwickeln. Hierzu gehören Unterrichtsmaterialien, die kulturbedingte Unterschiede oder Gemeinsamkeiten the- erweitern. Oft ist die fremde Kultur der Spiegel der eigenen und provoziert zur kritischen Auseinandersetzung bzw. Hinterfragung der eigenen Kultur"(Rademacher, 1991). Fremd erscheint zunächst all das, was nicht zur eigenen Gruppe, in der ein Individuum lebt, gehört; darüber hinaus auch das, was ausserhalb des eigenen Kulturkreises lebt oder existiert. Fremd wird oft negativ definiert. So ist im soziologischen Sinne ein Fremder Gruppe nicht angehört. Je homogener die Bezugsgruppe ist, desto stärker hebt sich der Fremde in der sozialen Wirklichkeit eines Kulturkreises ab und desto präziser ist auch der Begriff

\title{
DAS FREMDE UND DAS EIGENE: STEREOTYPEN UND FREMDSPRACHENUNTERRICHT
}

(1)

matisieren und veranschaulichen und die Rückwirkung auf das eigene Verhalten zulassen. Interkulturelle Kommunikation und Kompetenz schliessen auch die Fähigkeit ein, die eigene oder fremde Realität zu analysieren und "diese Ergebnisse in zwischenmenschlicher (d.h. auch interkultureller) Sprechweise mitteilen zu können". (Buttjes, 1982)

Dabei gehört Kultur zu jenen sozialwissenschaftlichen Begriffen, die für das Verständnis des Unbewussten von zentraler Bedeutung sind. Kultur und das Unbewusste in der Kultur stehen im unmittelbaren Zusammenhang mit dem Fremdbild und dem

Revista llustrada Maqueta y portada de John Heanffield
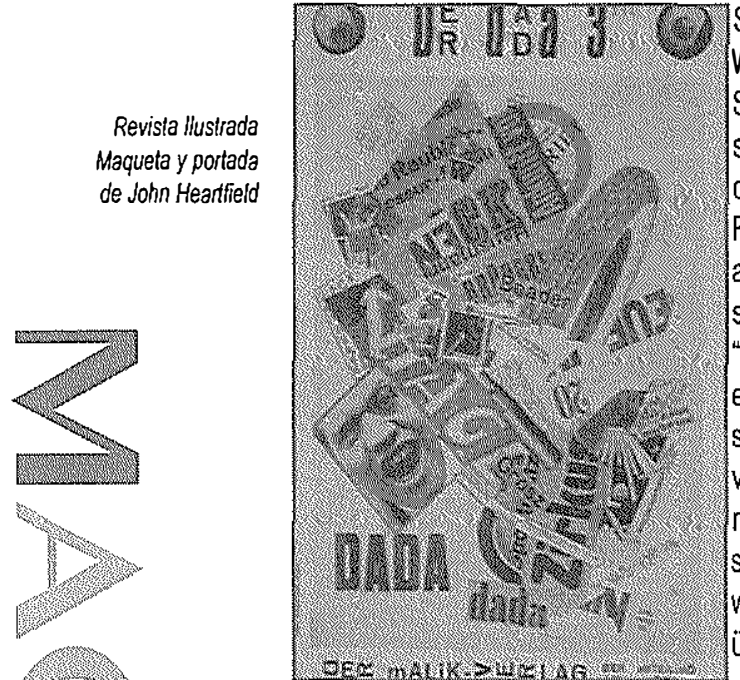
Selbstbild und der Wechselseitigkeit des Selbst- und Fremdverstehens. Die Auseinandersetzung mit dem Fremden ist gleichzeitig auch eine Auseinandersetzung mit dem Selbst: "Die Beschäftigung mit einer fremden Kultur setzt zwangsläufig voraus, sich mit der eige-

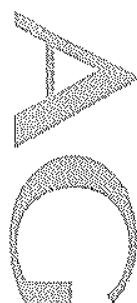
nen zu beschäftigen, sich dieser bewusst zu werden, sein Wissen über die eigene Kultur zu "fremd" gegenüber einem Aussenstehenden. Das Fremde kann demgegenüber aber auch faszinierend wirken. Die Faszination des Fremden kann zum Ausbruch aus dem bisherigen Umfeld führen und wirkt positiv auf die kulturelle Entwicklung.

Die Erfahrung von Fremde führt zum Verständnis des Anderen und kann es im optimalen Fall zum Eigenen machen. Aber ebenso kann die Erfahrung von Fremde die Inversion des Eigenen auslösen, dadurch kommt es zur Fremdwerdung des Eigenen und zur kritischen Distanzierung des vertrauten Eigenen. Das Eigene von aussen gesehen, bedeutet: um das Eigene erkennen zu können, muss man es verfremden. Um das Fremde verstehen zu können, muss man versuchen, ihm näher zu kommen; es gilt dann als verstanden, wenn es in die vertrauten Kategorien übersetzt wird.

Fremderfahrung steht somit im unmittelbaren Zusammenhang mit Eigenerfahrung. Die Auseinandersetzung mit dem Fremden aus dieser Perspektive stellt die Grundlage jeder Kulturvermittlung und auch die Basis jeder sozialen Orientierung dar. Neuerungen auf kulturellem Gebiet wären ohne das Einwirken und das Akzeptieren oder Nichtakzeptieren des Fremden kaum möglich: "Man könnte das formell auswerten: je offener ein gesellschaftliches oder kulturelles System, als desto wandlungs- und veränderungsfähiger dürfte es sich enweisen, je geschlossener, als desto autoritätsgebundener, homogener und traditionsgläubiger" (Bausinger,1977). 
Das Fremde ist allgegenwärtig präsent. Leben und Lernprozesse sind bestimmt durch die Begegnung mit dem Fremden. In einem Kulturkreis wird alltäglich Fremdheit erlebt, nicht nur der menschliche Verstand, seine Gefühle, sondern auch seine Sprache und sein Sprachvermögen werden davon beeinflusst. Eine Kultur lernt von der anderen und grenzt sich zugleich von ihr ab. Das Fremde wird so zum Ferment der Kulturentwicklung. Durch Interkulturalität soll die ethnozentrische Isolierung überwunden werden, kulturelle Unterschiede

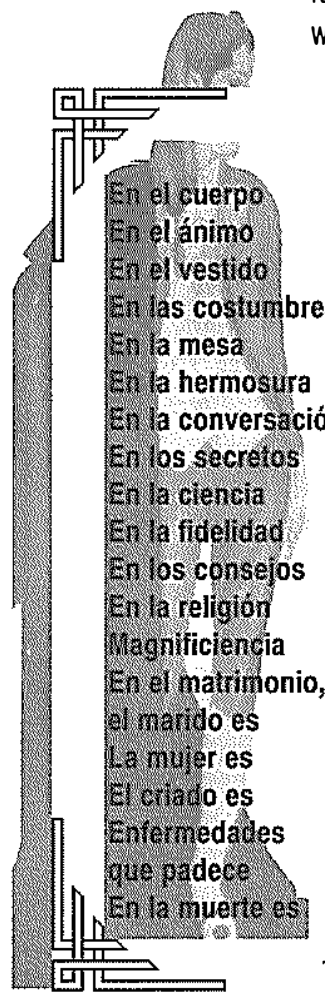

$\begin{array}{llll}\text { Alemán } & \text { Español } & \text { ltaliano } & \text { Francés } \\ \text { Robusto } & \text { Horrendo } & \text { Débil } & \text { Ágil } \\ \text { Oso } & \text { Elefante } & \text { Zorra } & \text { Águila } \\ \text { Mono } & \text { Modesto } & \text { Lúgubre } & \text { Proteo } \\ \text { Serio } & \text { Grave } & \text { Fácil } & \text { Ostentador } \\ \text { Ebrio } & \text { Fastidioso } & \text { Sobrio } & \text { Delicado } \\ \text { Estatua } & \text { Demonio } & \text { Hombre } & \text { Mújer } \\ \text { Aúlla } & \text { Habla } & \text { Delira } & \text { Canta } \\ \text { Olvidadizo } & \text { Mudo } & \text { Taciturno } & \text { Hablador } \\ \text { Jurista } & \text { Teólogo } & \text { Arquitecto } & \text { Algo de todo } \\ \text { Fiel } & \text { Falaz } & \text { Sospechoso } & \text { Ligero } \\ \text { Tardo } & \text { Cauto } & \text { Sutil } & \text { Precipitado } \\ \text { Supersticioso } & \text { Constante } & \text { Religioso } & \text { Celoso } \\ \text { En las fortificaciones } & \text { En las armas } & \text { En los templos } & \text { En los palacios } \\ & & & \\ \text { Señor } & \text { Tirano } & \text { Carcelero } & \text { Compañero } \\ \text { Alhaja doméstica } & \text { Esclava } & \text { Prisionera } & \text { Señora } \\ \text { Compañero } & \text { Sujeto } & \text { Obsequioso } & \text { Criado } \\ & & & \\ \text { Gota } & \text { Todas } & \text { Peste } & \text { Infección venérea } \\ \text { Desembarazado } & \text { Generoso } & \text { Desesperado } & \text { Violento }\end{array}$

Tabla del Alma de las Naciones más importantes de Europa del clérigoalemán J. Zahn (citada por Feijó sollen respeken Mapa intelectual. Finales siglo XVIII)

tiert werden und ihre Erkenntnis zum besseren Verständnis der eigenen und der fremden Kultur genutzt werden.

Welche Rolle spielen in dieser Thematik nun "Stereotypen"? Um dem mehr auf den Grund zu gehen, scheint es erforderlich den eigentlich klaren Begriff Stereotyp näher zu untersuchen:

\section{1 "Versuch" einer Begriffsdefinition}

Der ursprünglich aus der Buchdruckersprache stammende Ausdruck "Stereotyp": "Die Starrheit und Unveränderlichkeit der nach der Mater aus einem Stück gegossenen Druckplatte; die Unmöglichkeit, auf diese Platte nachtäglich Korrekturen anzubringen" (Manz,1968), wurde von Lippmann (1949) sozialwissenschaftlich definiert und versucht in eine kognitive Theorie einzubinden. In seiner Studie verdeutlicht er, dass es eine Diskrepanz zwischen den inneren Vorgängen des Wahrnehmens und Den- kens und den äusseren Vorgängen in der Umwelt gibt. Innere Vorstellungen und die äussere Welt müssen nicht übereinstimmen, wobei aber die Bilder und Schemen in unserem Kopf das Verhalten im sozialen Umfeld stärker beeinflussen als die herrschenden Bedingungen. Die Tatsachen, die gesehen werden, sind abhängig vom jeweiligen Standort und den Gewohnheiten der Augen. Ein nicht bekannter Standort, eine fremde Sprache können überraschend wirken, sofern die äusserlich herrschenden Bedingungen wirklich neu und
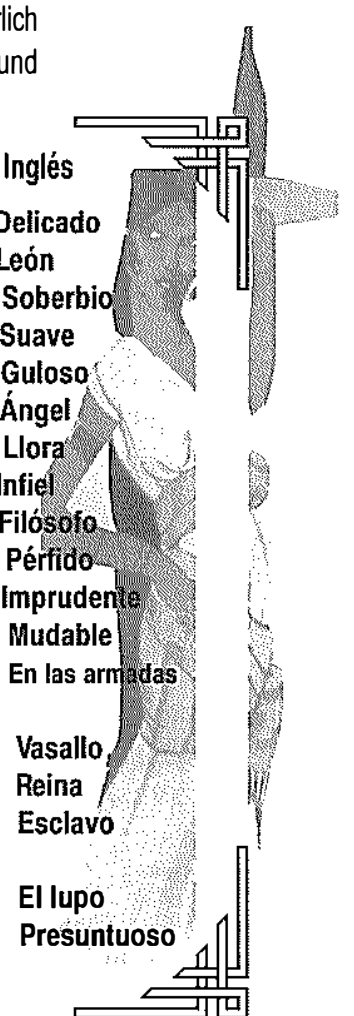

eigentümlich

sind. Menschen, die einer nicht bekannten ausländischen Gruppe angehören, können für einen aussenstehenden Betrachter gleich aussehen. Nur jeweils hervortretende Besonderheiten an Z.B. Farbe oder Grösse können von ihm unterschieden werden. Sollten einzelne Besonderheiten an Bedeutung gewinnen, so werden diese oft hervorgehoben, definiert und einer bestimmten Gruppe zugeschrieben. Aus der äusseren Welt, so Lippmann, wird das ausgewählt, was die jeweilige Kultur bereits definiert hat, nur das wird wahrgenommen, was in der Gestalt ausgewählt wurde, die die Kultur für seine Mitglieder stereotypisiert hat. Das Stereotyp steht hier als Ausdruck kulturell geprägter Konzepte, die der einzelne einer Gruppe übernimmt und danach die äussere Umgebung wahrnimmt und entsprechend interpretiert. Diese vorgefertigten Stereotypen sind nicht aufgrund eigener Erfahrungen entstanden; der jeweilige Träger solcher Prägungen kann durch sein vorgeformtes Denken seine Umwelt nur noch typisiert und reduziert in stereotypen Bildern wahrnehmen.

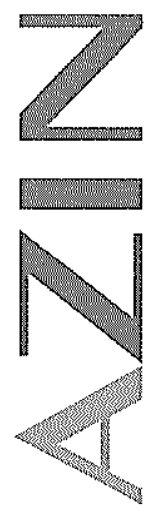


Der Begriff Stereotyp beinhaltet viele im politischen und gesellschafilichen Alltagsleben bedeutsame Sachverhalte, so dass eine grosse Vielfalt an sozialen Prozessen definiert werden kann. Quasthoff (1973) geht davon aus, dass es sich bei Stereotypen, dessen Begriffsbedeutung nach Lippmann von der Sozialpsychologie übernommen wurde, um eine sozialpsychologische Erscheinung handelt. Stereotypen stehen in enger Beziehung zu einer sprachlichen Äusserung und können sich sprachlich manifestieren. Hiernach ist primär sogar von einem sozialpsychologischen Problem auszugehen. Quasthoff stellt fest, dass der Begriff Stereotyp oft als Adjektiv oder Adverb gebraucht wird, um monotone sinnentleerte Wiederholungen zu beschreiben.

Quasthoff wie auch Manz (1968) beziehen sich auch auf die Arbeiten von Katz und Braly (1933 und 1935) deren Studien für die Stereotypenforschung von zentralerBedeutung sind. Der Stereotypbegriff wurde von beiden operationalisiert und legte somit die Grundlage für die eigentliche Stereotypenforschung in der Sozialpsychologie. Quasthoff stellt fest, dass beide Stereotyp als Überzeugung definierten. Der Begriff Überzeugung wird auch von Allport (1971) übernommen, für inn ist das Stereotyp eine "überstarke Überzeugung". Für Wenzel (1978) sind Stereotypen hingegen der verbale und bildhafte Ausdruck einer Vorstellung von einer sozialen Gruppe und dienen den einzelnen Individuen als Identifikation mit einer solchen Gruppe. Für Hofstätter (1959) stellen Stereotypen Leitbilder dar, die eine Erwartungshaltung gegenüber einer Gruppe von Menschen ausdrücken können und eine betont gruppenbezogene Bedeutung haben.

Festzustellen ist, dass der Begriff Stereotyp in seinen vielfältigen Definitionen schwer zu systematisieren ist. Eine grobe Einteilung ergibt, dass das Stereotyp als Überzeugung und auch als Urteil oder bildhafte Vorstellung klassifiziert wird. Aus diesem Grund wird Stereotyp von Quasthoff wie folgt definiert: "Ein Stereotyp ist der verbale Ausdruck einer auf soziale Gruppen oder einzelne Personen als deren Mitglieder gerichteten Überzeugung. Es hat die logische Form eines Urteils, das ungerechtfertigt in vereinfachender und generalisierender Weise, mit emotional wertender Tendenz, einer Klasse von Personen bestimmte Eigenschaften oder Verhaltensweisen zu- oder abspricht. Linguistisch ist es als Satz beschreibbar".

\section{Aspekte zur Stereotypenforschung und weiteren "Begrifflichkeiten"}

Den geschichtlichen Hintergrund der Stereotypenforschung betrachtend, lässt sich feststellen, dass er ursprünglich eher im amerikanischen Raum zu finden ist, nicht nur basierend auf der Einführung des Stereotypbegriffs durch Lippmann, sondern auch durch die in den USA nicht nur in der Vergangenheit herrschenden Einwanderungs- und Rassenprobleme. Diese Problematik, mit der sich die USA konfrontiert sah, sollte gelöst werden. Ame- rikanische Psychologen glaubten an den Erfolg und die Wirksamkeit der Wissenschaft und versuchten, mit der Stereotypenforschung ein Mittel zu finden, die Menschen von ihren Vorurteilen zu heilen. Man glaubte, dass Stereotypen feste, von sich aus kaum einem Wandel unterworfene Vorurteile sind. Durch positive Beeinflussung und Erziehung sollte die Irrationalität von stereotypen Vorstellungen bewusstgemacht werden. Da man aufgrund des wissenschaftlichen Standes der Forschungen schon früh zu der Erkenntnis gekommen war, dass Stereotypen das Handeln und Sozialverhalten der Menschen normativ und konstant steuern, entstand die Frage nach dem Zusammenhang zwischen negativen Einstellungen bzw. "rassischen" Vorurteilen und dem Begriff Stereotyp. Hiernach wurde dann folglich von rassischen Stereotypen ausgegangen. Nicht nur aus diesem Grund, sondern auch in Bezug auf die Auswirkungen von rassischen Vorurteilen im Dritten Reich, gewannen die wissenschaftlichen Untersuchungen über Stereotypen immer mehr an Bedeutung. Die damalige Wissenschaft ging von einem Stereotypenwandel aus, der sich mit der Theorie der Wandlungsprozesse beschäftigte. In der heutigen Stereotypenforschung geht man diesbezüglich von einem nationalen bzw. ethnischen Stereotyp aus. Die soziale Umwelt wird von einzelnen Mitgliedern, die selbst zu einer Gruppe gehö-

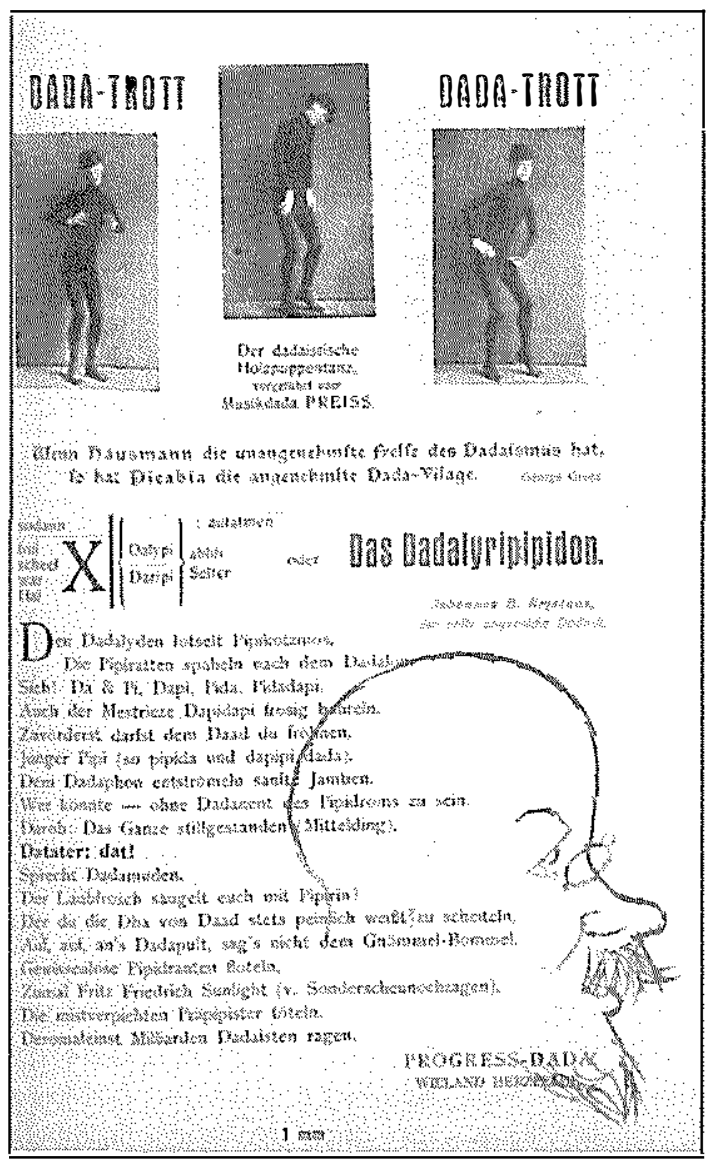


ren, in vielen Gruppen eingeteilt. Dabei wird ein Gruppenmitglied aufgrund seiner Nationalität oder seiner Gruppenzugehörigkeit charakterisiert. Aufgrund der psychologischen Auffassung, dass Stereotypen u.a. Kristallisierungspunkte gefühlsbetonter Einstellungen sind, lag hier nicht nur in der Vergangenheit oftmals das Gewicht der Stereotypenforschung auf den Begriffen Haltung und Einstellung. Schwer bestimmbare Einstellungen werden hier als analysierbare Stereotypen definiert. Ferner diente die Stereotypenforschung als Methodik und Ansatzpunkt zur Definition von Einstellung und wurde als neugewonnener Begriff ebenso in der Einstellungsforschung angesiedelt. Hier sind Stereotypen psychologische Vereinfachungen, die in Einstellungen und Meinungen enthalten sind. Im sozialpsychologischen Sprachgebrauch werden Gefühle, die man gegenüber von Gruppen hat, auch als soziale Einstellungen definiert. Dabei können sie Tendenzen mit positiv oder negativ wertendem Charakter sein. Quasthoff bemerkt zu Einstellung, dass der Begriff unscharf und umgangssprachlich verwendet wird, obwohl die jeweilig getroffenen Definitionen theoretisch ausgereifter scheinen: "Eine Einstellung ist eine allgemeine Disposition, Reaktionsbereitschaft, Gerichtetheit, also etwas Virtuelles, das erst durch seinen steuernden Einfluss in bestimmten Situationen gegenüber bestimmten Objekten wirksam und fassbar wird" (Quasthoff 1973).

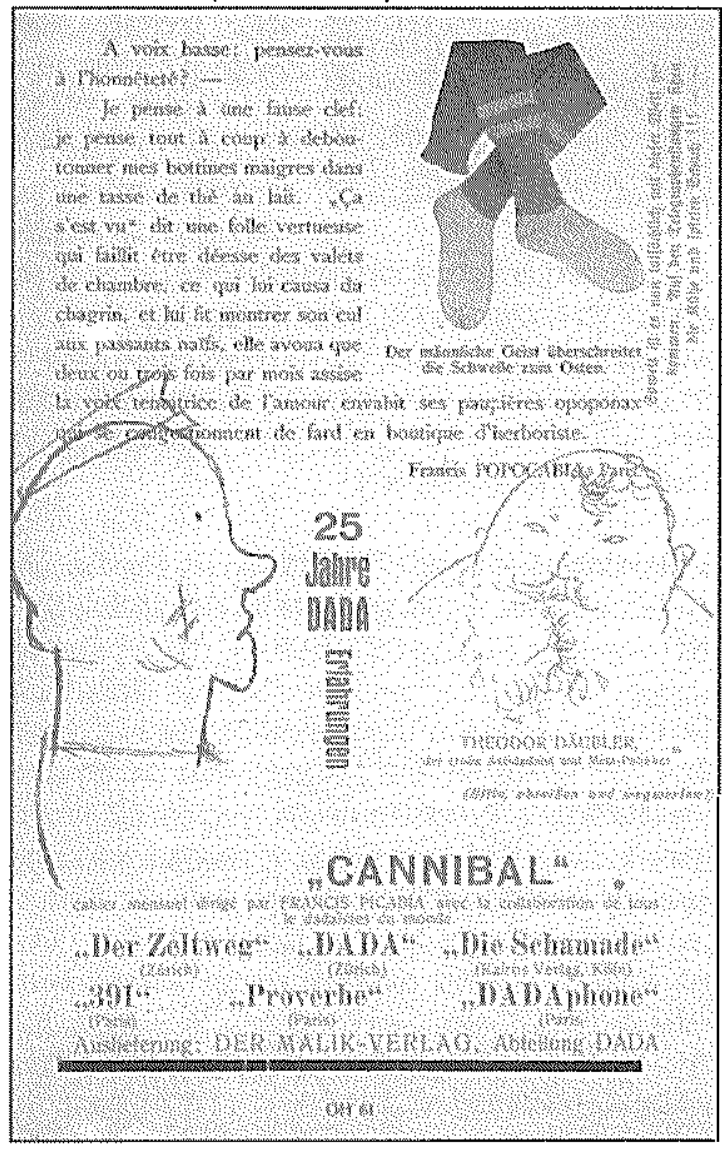

Sie unterscheidet an dieser Stelle Einstellung und Überzeugung und bezieht sich auf die von Allport getroffenen Definitionen. Er machì den Unterschied durch zwei Sätze deutlich:" Ich kann Schwarze nicht leiden": hier wird als Einstellung eine allgemeine Abneigung zum Ausdruck gebracht. Im zweiten Satz "Schwarze riechen schlecht" handelt es sich um eine Überzeugung mit behauptendem Charakter in Bezug auf die soziale Gruppe "Schwarze" und deren vermeintlichen Eigenarten. Den Unterschied zwischen Einstellung und Überzeugung definiert Quasthoff wie folgt: "Während eine Einstellung also lediglich eine affektive Haltung gegenüber dem Bezugsobjekt bezeichnet, bezeichnet eine Überzeugung ihren Gegenstand inhaltlich Qualitäten zu oder ab"(Quasthoff 1973). In einer später durchgeführten Untersuchung zur Abgrenzung der Begriffe wird von ihr die enge Verbindung von Stereotypen als linguistischem Gegenstand und der stereotypen Überzeugung als sozialpsychologischem Gegenstand dargestellt. Die Sozialpsychologie setzt hier die stereotype Überzeugung mit einer wertenden Einstellung gegenüber einer Gruppe in Verbindung. Einstellungen sind auch Bewertungen in Bezug auf eine Gruppe, während stereotype Überzeugungen diese Gruppen mit bestimmten Eigenschaften versehen. An dieser Stelle werden die folgenden Beispiele genannt: "Ich mag keine Schwarzen", ist hier also der Ausdruck einer Einstellung. "Schwarze sind dumm, faul und kriminell" stellt demgegenüber ein Stereotyp dar. Hier wird entsprechend eine stereotype Überzeugung mit Stereotyp gleichgesetzt. Wobei stereotype Überzeugungen psychologisch gesehen also Rationalisierungen für Einstellungen sein können. Aus dem Beispiel zwei, "Schwarze sind dumm, faul und kriminell" als stereotype Überzeugung kann im psychologischen Sinne der Schluss einer Rechtfertigung einer feindlichen Einstellung oder ablehnenden Verhaltens gegenüber o.g. Gruppe gezogen werden. Einstellungen und Verhalten gegenüber Gruppen oder einzelnen Mitgliedern von Gruppen werden in der Psychologie durch stereotype Überzeugungen begründet. Benachteiligungen und Diskriminierungen sind stereotype Überzeugungen im negativen Sinne. Diese spezifischen Verhaltensformen beeinflussen nicht nur die sogenannten Randgruppen oder sozialen Minoritäten einer sozialen Gruppe, sondern auch die Beziehungen der Nationen und deren politische Verhältnisse. Den gesellschaftlichen Kontext betrachtend, lässt sich feststellen, dass sich nicht nur die ökonomischen und politischen Verhältnisse eines Landes verändern, sondern auch die stereotypen Überzeugungen in Bezug auf die Minoritäten einer jeweiligen Nation. Also sind es nicht nur die stereotypen Überzeugungen, die entsprechende Verhaltensformen auslösen: die von einer Gesellschaft aus gesehenen Verhaltensformen mit ideologischem Charakter in Bezug auf eine im Moment erscheinende und ausgewählte Randgruppe können diese stereotypen Überzeugungen auslösen.

Bei dem Begriff Einstellung geht es um Objekte wie Fremdgrup-
Páginas interiores de la Revista con ilustiaciones de George Grosz
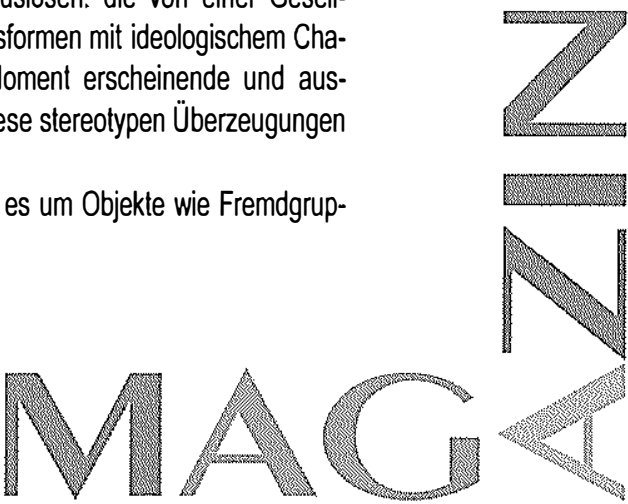
pen und Nationalitäten. Wird von einer Person einer Gruppe durch seine vorhandene Bereitschaft ein bestimmtes psychologisches Objekt immer wieder in einer bestimmten, wiederkehrenden Art und Weise erfasst und wird entsprechend gleich darauf reagiert, so kann man von einer Einstellung ausgehen. Eine Meinung hingegen bezieht sich auf bestimmte Fragen. Sie kann aber von verschiedenen Einstellungen begründet werden, wie umgekehrt aus ähnlichen oder gleichen Einstellungen verschiedene Meinungen entstehen können. Einstellungen sind nach Quasthoff virtueller Natur. Wie auch Meinungen und Überzeugungen als Bewusstseinsinhalte, sind sie nur über ihre Äusserungsform zu erfassen

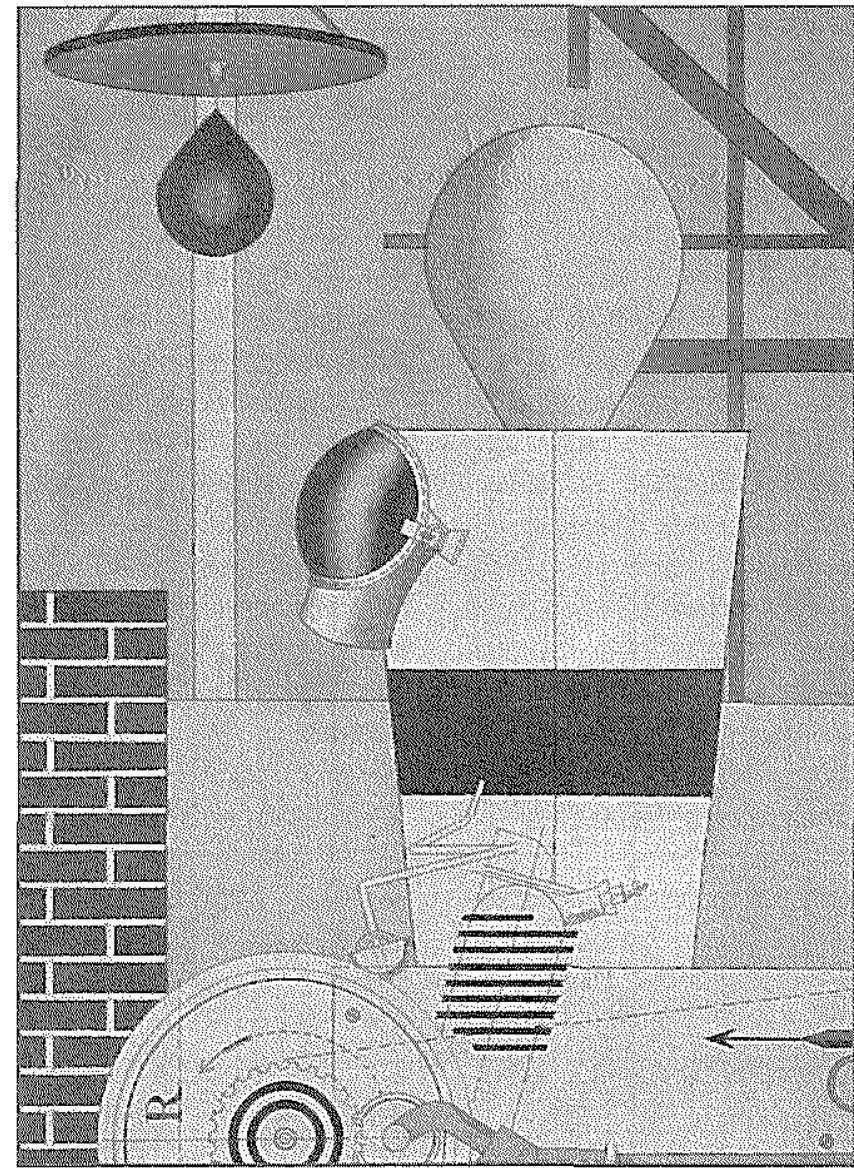
und zu beschreiben. Will man Überzeugung und Meinung vergleichen, so ist festzustellen, dass Überzeugungen dauerhafter und konsistenter sind, während Meinungen punktuell und abhängig von der jeweiligen spezifischen Situation sind. Abgesehen von der Abgrenzung zu Einstellung, besteht kaum ein Unterschied zwischen Überzeugung und Meinung.

Auch der Begriff "Image" findet seine Bedeutung in der Stereotypenforschung. Neben Stereotyp und Vorurteil wird er oft für die selben Erscheinungen gebraucht.. Der von dem psychoanalytischen "Imago" abgeleitete Begriff wird oft synonym zu Stereotyp gebraucht: die bildliche Darstellung von einem psychologischen Objekt. Dröge (1967) leitet Image von der lateinischen Bedeutung "Bild" ab, wobei er Image als nicht dauernd und kurzlebig beschreibt. Stereotypen hingegen sind Vorstellungen, die "natürliche bildhafte Elemente enthalten" (Dröge1967). Stereotyp wird hier mit Verhaltenserwartungen von einzelnen Individuen und deren Gruppen definiert, während Image "die Vorstellungsbilder von Konsumgütern belegt" (Dröge 1967). Bei Image geht es um die Beziehung zwischen Subjekt und Objekt und deren unterschiedlicher Dialektik der Bewusstseinsbezüge im Vergleich zu Stereo- typ, wo sich ein Subjekt auf ein anderes Subjekt bezieht. Bei Image steht die dingliche Welt im Vordergrund. Der einzelne Gegenstand wird geschätzt, weil er gebraucht wird oder weil er gebraucht werden kann. Dieser Gegenstand wird mit einem Konsumbild versehen. Das einzelne Individuum eignet sich dann weniger den Gegenstand an, sondern eher das daraus enstandene Leitbild (=Image). Image enthält demnach auch Stereotypen, da es um die Harmonisierung dieser dringlichen Welt geht. Durch das Anpassungsstreben der Individuen innerhalb einer Gruppe wird Image zielorientiert und ist somit eine "objektgerichtete Verhaltensbestimmung" (Dröge 1967) mit daraus resultierenden sozialen Zwängen. Auch Quasthoff verweist auf die oft als synonym gebrauchten Begriffe Image und Stereotyp. Hier steht auch das Eigeninteresse eines Individuums an seinem Image in der jeweiligen Gruppe im Vordergrund. Ein Stereotyp kann in diesem Zusammenhang ein Image sein, wenn es als Bezugsobjekt einer Gruppe zugeschrieben wird.

Bei dem Begriff Klischee geht es ebenfalls um den Objektbereich. Wie bereits erwähnt, bezieht sich Image auf das Verhältnis Subjekt - Objekt und Stereotyp auf Subjekt - Subjekt. Ein Klischee hingegen kann sich unbegrenzt auf alles beziehen. Hierbei spielt der fast nicht vorhandene Überzeugungscharakter eine grosse Rolle, denn ein Klischee stellt Ausdrucksweisen dar, die ohne eine Überzeugung übernommen werden. Klischees sind sinnentleerte Konzepte und äussern sich durch unbedacht geäusserte Rede- und Denkschemata und vorgeprägte Bilder und Wendungen. Diese Darstellungsweisen eines möglichen Ausdrucks können, insofern sie verbal dargestellt werden, als stereotyper Gebrauch definiert werden, wenn hier unter Stereotyp feststehend, gleiche und ständig wiederkehrende Ausdrücke und Wendungen verstanden wird. 
Der wohl am meisten mit Stereotyp als synonym gebrauchte Begriff ist das Vorurteil. Vorurteile über bestimmte Gruppen von Menschen wie z.B. "Türken stinken", oder "Arabern kann man nicht trauen" sind allgemein bekannt und lassen ihren negativen Charakter leicht erkennen. In den Bereichen der Psychologie und der Soziologie werden diese als gesellschaftlich bedingte Erscheinungen erklärt. In der Vorurteilsforschung werden Vorurteile als kognitive Elemente und als Bewusstseinskategorien angesehen. Vorurteile als kognitive Kategorien oder individuelle Vorstellungsbilder beziehen sich auf soziale Gruppen, die an bestimmte Wertvorstellungen gebunden sind. Nach Estel (1983) sind Vorurteile als soziale Kategorisierungen Bestandteil der Kultur und dienen wie auch andere kulturelle Elemente dem Individuum zur Orientierung und zum Handel in einer jeweiligen Gruppe. Hierbei kann das Vorurteil eine Alibifunktion haben, um sich in eine entsprechende Gruppe zu integrieren. Es dient zur Aufrechterhaltung der sozialen Ordnung und zur Herstellung und Bewahrung kollektiver Identität. Somit besteht ein gewisses Angewiesensein auf eine Vorurteilsbereitschaft für die eigene Sozialisierung des Individuums in der Gruppe. Vorurteile sind Bestandteile des normativen Wertesystems bei der Integration einer jeweiligen Gruppe, wobei "das Welt- und Gesellschaftsbild, das eine Gruppe welcher Art auch immer so entwickelt mit seiner Wertordnung und seinem Normensystem - mit einem Wort: Die Ideologie dieser Gruppe hat für die Enstehung und Existenz dieser Gruppe eine wesentliche konstitutive Bedeutung" (Estel 1983).

Die Wirklichkeit einer Gruppe wird aus deren kulturellen Werteund Normensystem gebildet, hierbei bilden Vorurteile kognitive Muster, die miteinander geteilt werden und an denen sich die Mitglieder dieser Gruppe orientieren und nach denen sie handeln. Die oft in der Vorurteilsforschung festgestellte "Starrheit" bei Vorurteilen und der damit verbundenen selbstbestätigenden Wirkung findet somit ihre Begründung in der Soziologie. Durch diese Ansätze zur Erklärung der Vorurteile werden in der Vorurteilsforschung auch die Ansätze zur Klärung von Rassenbeziehungen gefunden. Denn innerhalb des Rassismus geht es um eine bestimmte Form von Vorurteilen. Spezifisch bei diesen Rassenvorurteilen ist von Elementen einer Kultur und folglich auch von deren jeweiligen Ideologie auszugehen. Das Vorurteil ist "ein Teil der Kultur und muss dementsprechend verstanden werden"(Estel 1983). In der heutigen Gesellschaft produzieren einzelne Gruppen mit gleichen Interessen immer neue Images, die je nach ihrer Geltungskraft variieren und von Urteilen zu Vorurteilen werden können. Individuell kategorisierende Urteile über Menschen sind also Vorurteile; diese Urteile weisen einzelne Stereotypen auf, die oft eine negative Bewertung in Bezug auf die Beziehung des Urteilenden zu einer bewertenden Gruppe erkennen lassen. Je stärker einzelne Merkmale eines Bezugsobjekts zutreffen und je mehr sie an Gültigkeit gewinnen, umso deutlicher wird der stere- otype Charakter. Gemäss der Vorurteilsforschung, die Vorurteil und Stereotyp immer wieder voneinander trennt, sind Vorurteile Urteile über die Umwelt, die ohne eine Erfahrung getroffen werden, handlungbeeinflussend wirken können und ihren Ausdruck in Stereotypen finden. Vorurteile werden weiterhin als negative Einstellung gegenüber Gruppen definiert, die auf negativen Stereotypen beruhen können. Stereotypen können somit Komponenten von Vorurteilen sein. Ähnlich wie Estel sieht auch Quasthoff das Vorutteil als neutrale kognitive Kategorie, die der Orientierungsfunktion dient. In Bezug auf ein Bezugsobjekt lassen sich ihrer Meinung nach allerdings Vorurteil und Stereotyp voneinander trennen.

In der Sozial psychologie wird das Vorurteil als soziales Vorurteil verstanden, das sich auf Gruppen oder auf einzelne Mitglieder dieser Gruppen richtet. Vorurteile setzen sich aus Einstellungsund Überzeugungsfaktoren zusammen, fraglich ist, ob das für Stereotypen ebenso zutriffi, oder ob Stereotypen lediglich eine Bezeichnung für eine Überzeugung ist. Eine Unterscheidung der Begriffe und Überzeugung als Komponenten für Vorurteil ist ihrer Meinung nach nicht einzusehen, denn in beiden "ist ein VorausUrteil über eine gesammte Gruppe von Menschen begründet, das in jedem Fall wertender Natur ist, unabhängig davon, ob der Einstellungs- und Überzeugungsfaktor überwiegt"(Quasthoff 1973). Überzeugungen und Einstellungen bilden das soziale Vorurteil, wobei Einstellung eine Disposition ist. Einstellung und Überzeugung sind voneinander abhängig, da sie durch eine bestimmte Art psychologisch miteinander verknüpft sind, daraus ergibt sich, "dass die spezifische Form der Überzeugung innerhalb des sozialen Vorurteils ebenfalls dispositionellen Charakter hat und realisiert werden muss, um erkennbar zu sein" (Quasthoff 1973).

Zusammenfassend kann also gesagt werden, dass Vorurteil und Stereotyp ein Voraus-Urteil sein können. Vorurteile beinhalten die Kategorie Einstellung, die eine allgemeine Haltung zwischen Abneigung und Sympathie bezeichnet, sowie die Kategorie Überzeugung, die ihrem Objekt Qualitäten zu- oder abspricht und sehr häufig Rationalisierungen für Einstellungen darstellen. Das Stereotyp stellt hingegen die verbale Äusserungsform von Überzeugungen dar, die sich auf einzelne Gruppen beziehen.

J.D.

5

Bibliographie im Anschluss an Teil 2 dieses Artikels im mAGAzin $n^{\circ} 6$

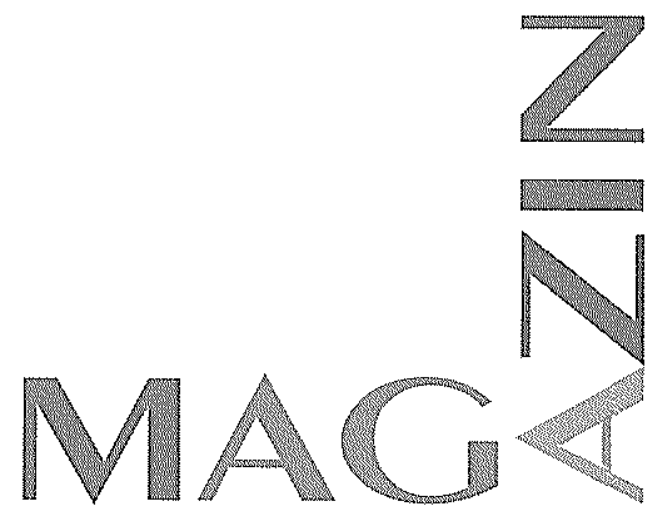

